\title{
ARCHITECTURE FOR FILTER ALGORITHM IN GPS/INS INTEGRATION
}

\author{
Ahmed Azouz* and Ahmed E. Abdalla*
}

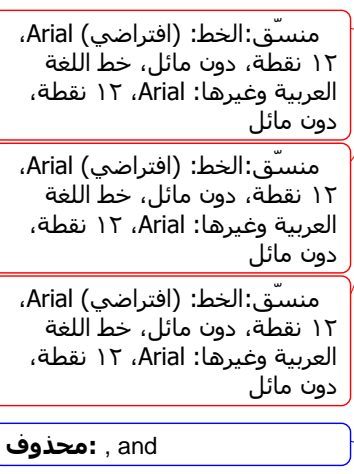

\begin{abstract}
An inertial navigation system (INS) exhibits relatively low noise but tends to drift ol time. In contrast, Global Positioning System (GPS) errors are relatively noisy, ! exhibit no long-term drift. Integrated INS/GPS navigation systems provide the best both worlds: the low short term noise characteristics of INS and the long term stabi of GPS are combined to provide a navigation solution with accuracy, reliability a robustness far beyond the sum of the constituent parts. However, in order to fl evaluate the performance of an integrated INS/GPS system, it is necessary stimulate both the GPS and inertial components of the system simultaneously. In tl paper, the architectures for common filter algorithms in GPS/INS loose integrati are presented. The error dynamics for attitude calculation are derived. Algorithı based on quaternions and direction cosines are used. Simulation results : analyzed to evaluate the different systems. This system evaluation are required help to create specification data; to aid integration algorithm design and tuning; determine if the receiver meets a given specification; to create conditions beyo those which can be created during live trials; and to recreate a known anomaly whi occurred in the real world.
\end{abstract}

\section{KEYWORDS}

Inertial Navigation System, Inertial Measurement Unit, Global Positioning Systems Loose integration ,Direction Cosines Matrix, Skew-symmetric matrix, and Quaternions. 


\section{1- INTRODUCTION}

GPS and INS have complementary qualities that make them ideal to use for sens fusion. The limitations of GPS include occasional high noise content, outages wh satellite signals are blocked, interference, and low bandwidth. The strengths of $\mathrm{GI}$ include its long-term stability and its capacity to function as a stand-alone navigati system. In contrast, inertial navigation systems are not subject to interference outages, have high bandwidth and good short-term noise characteristics, but ha long-term drift errors and require external information for initialization. A combin system of GPS and INS subsystems can exhibit the robustness, higher bandwic and better noise characteristics of the inertial system with the long-term stability GPS. The level and complexity of GPS and INS coupling is dictated by seve factors, including desired navigation accuracy, quality of the inertial measureme unit (IMU), and required robustness of the GPS receiver outputs. The levels integration are usually classified as loose integration, tight integration and ultra-tic or deep Loose integration is the simplest method of coupling [1] where GPS and II generate navigation solutions independently (position, velocity and attitude). T Kalman filter used in GPS/INS integration module is independent on the Kalman fil of GPS module, which increases the reliability of the system in case of failure GPS INS.

Tight Integration is a more complex level of coupling is tight integration [2], where t raw GPS ephemeris information and the position and velocity from INS algoritl used to predict pseudoranges and Doppler measurement. The tight integrati method contains only a single Kalman filter.

Ultra-Tight Integration is the most complex level of coupling [3]. It occurs at the GI tracking-loop level. It takes the difference between predicted in In-phase a Quatrature-phase of INS and the raw of GPS measurements In-phase a Quatrature-phase to determine the error estimates of the position, velocity a attitude. In terms of performance, ultra-tight integration also offers the most benet in terms of accuracy and robustness improvements to the GPS receiver and ove system.

In this paper, the architectures for common filter algorithms in GPS/INS loo integration are presented. The lose integration is used due to its computatiol simplicity comparing with other integration algorithms. More over, it is suitable parallel processing.

The error dynamics for attitude calculation are derived. Algorithms based quaternions and direction cosines are used. Simulation results are analyzed explore the advantages of each algorithm.

\section{1- Architecture of Loose Integration}

GPS/INS system with. loose integration is depicted in Fig. , for which the operation steps are given as bellow:

1- The Kalman filter of GPS extracts the position velocity data processes the $r_{i}$ data received by GPS receiver.

2- The raw IMU measurements, (Specific forces and angular rates) are process through the INS algorithm to determine the position, velocity and attitude. 
محذوف: integration through Kalman

محذوف: , and

محذذوف: integration through Kalman

محذوف: , and

منسّق:مضبوطة، تباعد الأسطر: مفرد، السماح:مضبوطة بعلامات التنقيط الأسطر

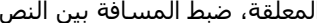

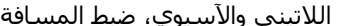
بين النص والأرقام الآسيوية، محاذاة الخط: تلقائي

محذوف: integration through Kalman

منسّق:الخط: (افتراضي) Arial، خط اللغة العربية وغيرها: (افتراض)، Arial،

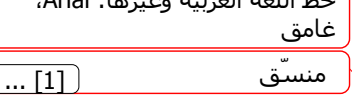

منسّق:الخط: (افتراضي) Arial ، مونة دون غامق، خط اللغة العربية (افتراضية

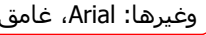

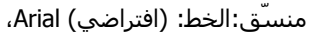

خط اللغة العربية وغيرها: (Arial،

منسِّق:الخط: (افتراضي) Arial، دون غامق، خط اللغطة العربية وغيرها: Arial، غخطامق

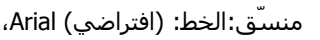
خط اللغة العربية وغيرها: Arial، غامق

: محذوف : 1
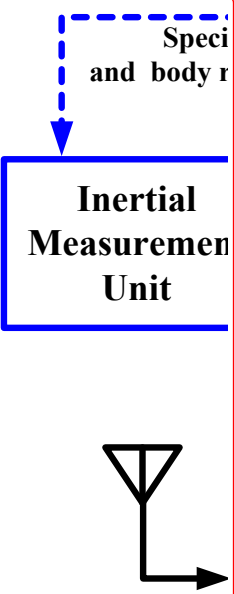

... [2] ग:

محذوف: , and محذوف: $F_{R R}, F_{R V}$
3- The GPS/INS Jntegration using Kalman filter takes the difference between $t$ position and velocity from GPS Kalman filter and INS algorithm to determine $t$ error estimates in position, velocity and attitude.

4- The error estimates from GPS/INS Integration using Kalman filter are feedback correct the position, velocity and attitude in INS algorithm.

- The loose integration method is distinguished by its simplicity in implementation a its robustness. If one of the sensors (INS or GPS) fails, a solution is still given by $t$ other sensor. Other advantage of the loose integration can be seen in the processi time of the algorithm due to generally smaller state vectors. One of the benefits loose integration is that the INS/GPS Integration using Kalman filters has better noi characteristics than the GPS solution alone.

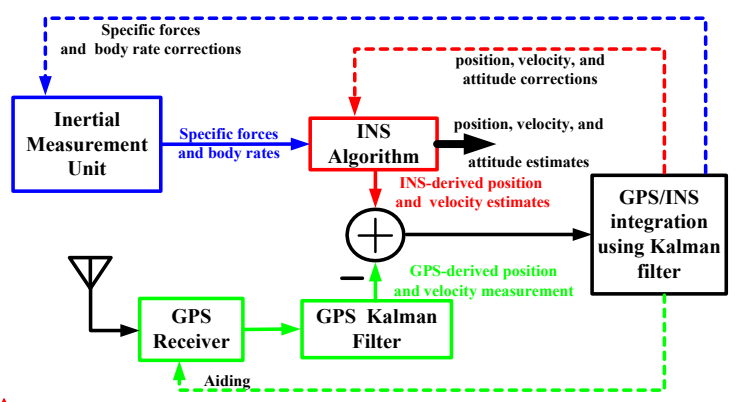

Fig.1. GPS/INS system with loose integration

The disadvantage is mainly that it is impossible to provide measurement update frc the GPS filter during poor GPS cover (less than four satellites) ${ }_{\text {L }}$ oose integration best implemented with higher quality inertial sensors (navigation-grade or tactic grade) if the GPS outages are long in duration. Lower quality inertial sensors c also provide some immunity against momentary GPS outages, especially if th various biases were calibrated using GPS prior to the outage. In general, lon quality inertial sensors are suited for applications where GPS outages are infreque and short in duration.

\section{2- POSITION ERROR DYNAMICS}

The error dynamics equations for positions in the $\mathrm{N}$-frame are functions of positi and velocity error $[4,5]$.

$$
\delta \dot{\mathrm{r}}^{\mathrm{n}}=\mathrm{F}_{\mathrm{RR}} \delta \mathrm{r}^{\mathrm{n}}+\mathrm{F}_{\mathrm{RV}} \delta \mathrm{v}^{\mathrm{n}}
$$

where: $\delta \mathrm{r}^{\mathrm{n}}=(\delta \varphi \delta \lambda \delta \mathrm{h})^{\mathrm{T}}$ is the error in position in $\mathrm{N}$-frame, $\delta \mathrm{v}^{\mathrm{n}}=\left(\delta \mathrm{v}_{\mathrm{N}} \delta \mathrm{v}_{\mathrm{E}} \delta \mathrm{v}_{\mathrm{D}}\right)^{\mathrm{T}}$ the error in velocity in $\mathrm{N}$-frame, $\mathrm{F}_{\mathrm{RR}}$ and $\mathrm{F}_{\mathrm{RV}}$ are described by the followi equations. 


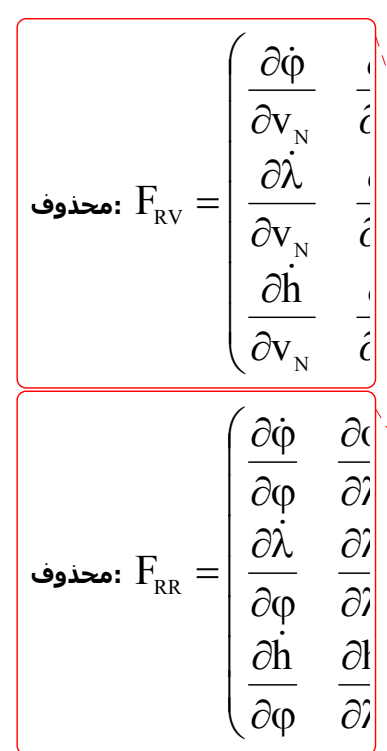
$\ldots[3]$

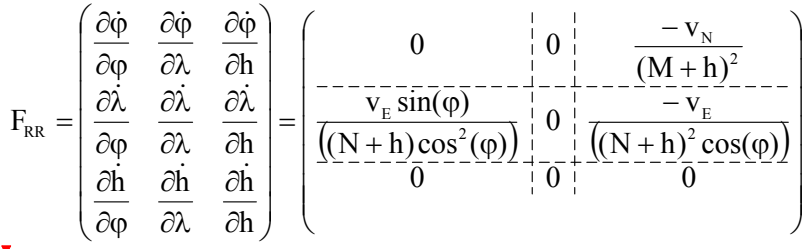

$$
\begin{aligned}
& \mathrm{F}_{\mathrm{RV}}=\left(\begin{array}{lll}
\frac{\partial \dot{\varphi}}{\partial \mathrm{v}_{\mathrm{N}}} & \frac{\partial \dot{\varphi}}{\partial \mathrm{v}_{\mathrm{E}}} & \frac{\partial \dot{\varphi}}{\partial \mathrm{v}_{\mathrm{D}}} \\
\frac{\partial \dot{\lambda}}{\partial \mathrm{v}_{\mathrm{N}}} & \frac{\partial \dot{\lambda}}{\partial \mathrm{v}_{\mathrm{E}}} & \frac{\partial \dot{\lambda}}{\partial \mathrm{v}_{\mathrm{D}}} \\
\frac{\partial \dot{\mathrm{h}}}{\partial \mathrm{v}_{\mathrm{N}}} & \frac{\partial \dot{\mathrm{h}}}{\partial \mathrm{v}_{\mathrm{E}}} & \frac{\partial \dot{\mathrm{h}}}{\partial \mathrm{v}_{\mathrm{D}}}
\end{array}\right)=\left(\begin{array}{ccc}
\frac{1}{(\mathrm{M}+\mathrm{h})} & 0 & 0 \\
0 & \frac{1}{((\mathrm{~N}+\mathrm{h}) \cos (\varphi))} & 0 \\
0 & 0 & -1
\end{array}\right)
\end{aligned}
$$

\section{3- VELOCITY ERROR DYNAMICS}

The velocity dynamics equation of inertial navigation system is [4]:

$$
\dot{\mathrm{v}}^{\mathrm{n}}=\mathrm{C}_{\mathrm{b}}^{\mathrm{n}} \mathrm{f}^{\mathrm{b}}-\left(2 \omega_{\mathrm{ie}}^{\mathrm{n}}+\omega_{\mathrm{en}}^{\mathrm{n}}\right) \times \mathrm{v}^{\mathrm{n}}-\mathrm{g}^{\mathrm{n}}
$$

Take in consideration the accumulation error, the calculation and measurement err The computed version of the velocity dynamics equation can be expressed as

$$
\dot{\overline{\mathrm{v}}}^{\mathrm{n}}=\overline{\mathrm{C}}_{\mathrm{b}}^{\mathrm{n}} \overline{\mathrm{f}}^{\mathrm{b}}-\left(2 \bar{\omega}_{\mathrm{ie}}^{\mathrm{n}}+\bar{\omega}_{\mathrm{en}}^{\mathrm{n}}\right) \times \overline{\mathrm{v}}^{\mathrm{n}}-\overline{\mathrm{g}}^{\mathrm{n}}
$$

$\rightarrow \dot{\overline{\mathrm{V}}}^{\mathrm{n}}=\dot{\mathrm{v}}^{\mathrm{n}}+\delta \dot{\mathrm{v}}^{\mathrm{n}} \quad$ where:

$\overline{\mathrm{C}}_{\mathrm{b}}^{\mathrm{n}}=\mathrm{C}_{\mathrm{b}}^{\mathrm{n}}+\delta \mathrm{C}_{\mathrm{b}}^{\mathrm{n}}$

$\overline{\mathrm{f}}^{\mathrm{b}}=\mathrm{f}^{\mathrm{b}}+\delta \mathrm{f}^{\mathrm{b}}$

$\bar{\omega}_{\mathrm{ie}}^{\mathrm{n}}=\omega_{\mathrm{ie}}^{\mathrm{n}}+\delta \omega_{\mathrm{ie}}^{\mathrm{n}}$

$\bar{\omega}_{\mathrm{en}}^{\mathrm{n}}=\omega_{\mathrm{en}}^{\mathrm{n}}+\delta \omega_{\mathrm{en}}^{\mathrm{n}}$

$\overline{\mathrm{v}}^{\mathrm{n}}=\mathrm{v}^{\mathrm{n}}+\delta \mathrm{v}^{\mathrm{n}}$

$\overline{\mathrm{g}}^{\mathrm{n}}=\mathrm{g}^{\mathrm{n}}+\delta \mathrm{g}^{\mathrm{n}}$

where: $\delta \mathrm{v}^{\mathrm{n}}$ is the error in velocity in $\mathrm{N}$-frame. ${ }_{-} \delta \mathrm{C}_{\mathrm{b}}^{\mathrm{n}}$ is the error in transformation $\mathrm{fr}$ B-frame to $\mathrm{N}$-frame. $-\delta \mathrm{f}^{\mathrm{b}}$ is the error in specific force measure in the B-fran $\delta \omega_{\mathrm{ie}}^{\mathrm{n}}$ is The error in projection of the rotating rate vector of the E-frame $\mathrm{W}$ respect to the l-frame on the $\mathrm{N}$-frame. $\delta \omega_{\mathrm{en}}^{\mathrm{n}}$ is The error in projection of $\mathrm{t}$ rotating rate vector of the $\mathrm{N}$-frame with respect to the E-frame on the $\mathrm{N}$-fran $\delta \mathrm{g}^{\mathrm{n}}$ is The error in gravitational acceleration.

By neglecting the second order error terms, Eq. (5) can be reduced to

$$
\delta \dot{v}^{n}=-\left(2 \delta \omega_{i e}^{n}+\delta \omega_{e n}^{n}\right) \times v^{n}-\left(2 \omega_{i e}^{n}+\omega_{e n}^{n}\right) \times \delta v^{n}-\delta g^{n}+C_{b}^{n} \delta f^{b}+\delta C_{b}^{n} f^{b}
$$


Eq. (6) can be reduced to

$$
\delta \dot{\mathrm{v}}^{\mathrm{n}}=\mathrm{F}_{\mathrm{VR}} \delta \mathrm{r}^{\mathrm{n}}+\mathrm{F}_{\mathrm{VV}} \delta \mathrm{v}^{\mathrm{n}}+\delta \mathrm{C}_{\mathrm{b}}^{\mathrm{n}} \mathrm{f}^{\mathrm{b}}+\mathrm{C}_{\mathrm{b}}^{\mathrm{n}} \delta \mathrm{f}^{\mathrm{b}}
$$

محذوف: $\mathrm{F}_{\mathrm{VR}}$ محذوف: , where: $F_{\mathrm{VR}}$ and $\mathrm{F}_{\mathrm{Vv}}$ described by the following equations [4].

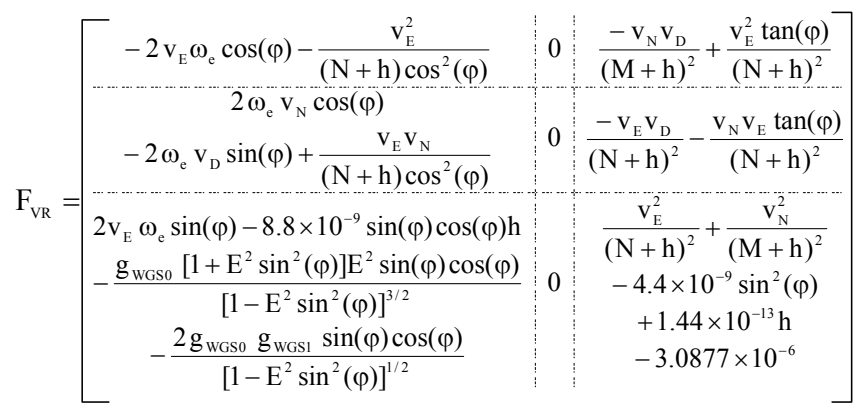

$$
\mathrm{F}_{\mathrm{VV}}=\left(\begin{array}{c:c:c}
\frac{\mathrm{v}_{\mathrm{D}}}{(\mathrm{M}+\mathrm{h})} & -2 \omega_{\mathrm{e}} \sin (\varphi)-\frac{2 \mathrm{v}_{\mathrm{E}} \tan (\varphi)}{(\mathrm{N}+\mathrm{h})} & \frac{\mathrm{v}_{\mathrm{N}}}{(\mathrm{M}+\mathrm{h})} \\
\hdashline 2 \omega_{\mathrm{e}} \sin (\varphi)+\frac{\mathrm{v}_{\mathrm{E}} \tan (\varphi)}{(\mathrm{N}+\mathrm{h})} & \frac{\mathrm{v}_{\mathrm{D}}+\mathrm{v}_{\mathrm{N}} \tan (\varphi)}{(\mathrm{N}+\mathrm{h})} & 2 \omega_{\mathrm{e}} \cos (\varphi)+\frac{\mathrm{v}_{\mathrm{E}}}{(\mathrm{N}+\mathrm{h})} \\
\hdashline(\mathrm{M}+\mathrm{h}) & -2 \omega_{\mathrm{e}} \cos (\varphi)-\frac{2 \mathrm{v}_{\mathrm{E}}}{(\mathrm{N}+\mathrm{h})} & 0
\end{array}\right)
$$

The calculation of term $\delta \mathrm{C}_{\mathrm{b}}^{\mathrm{n}} \mathrm{f}^{\mathrm{b}}$ and $\mathrm{C}_{\mathrm{b}}^{\mathrm{n}} \delta \mathrm{f}^{\mathrm{b}}$ depends on solution approach as given

\begin{tabular}{|c|c|}
\hline Algorithm & $\mathrm{C}_{\mathrm{b}}^{\mathrm{n}} \delta \mathrm{f}^{\mathrm{b}}$ \\
\hline $\begin{array}{l}\text { Quaternion } \\
\mathrm{s}\end{array}$ & $\begin{array}{l}\mathrm{C}_{\mathrm{b}}^{\mathrm{n}} \delta \mathrm{f}^{\mathrm{b}}=\mathrm{G}_{\text {AVQ }}\left(\begin{array}{l}\delta \mathrm{f}_{\mathrm{x}} \\
\delta \mathrm{f}_{\mathrm{y}} \\
\delta \mathrm{f}_{\mathrm{z}}\end{array}\right) \\
\text { where: } \mathrm{G}_{\text {AVQ }}=\left(\begin{array}{ccc}\mathrm{q}_{1}^{2}+\mathrm{q}_{0}^{2}-\mathrm{q}_{2}^{2}-\mathrm{q}_{3}^{2} & 2\left(\mathrm{q}_{1} \mathrm{q}_{2}-\mathrm{q}_{3} \mathrm{q}_{0}\right) & 2\left(\mathrm{q}_{1} \mathrm{q}_{3}+\mathrm{q}_{2} \mathrm{q}_{0}\right) \\
2\left(\mathrm{q}_{1} \mathrm{q}_{2}+\mathrm{q}_{3} \mathrm{q}_{0}\right) & \mathrm{q}_{2}^{2}+\mathrm{q}_{0}^{2}-\mathrm{q}_{1}^{2}-\mathrm{q}_{3}^{2} & 2\left(\mathrm{q}_{2} \mathrm{q}_{3}-\mathrm{q}_{1} \mathrm{q}_{0}\right) \\
2\left(\mathrm{q}_{1} \mathrm{q}_{3}-\mathrm{q}_{2} \mathrm{q}_{0}\right) & 2\left(\mathrm{q}_{2} \mathrm{q}_{3}+\mathrm{q}_{1} \mathrm{q}_{0}\right) & \mathrm{q}_{3}^{2}+\mathrm{q}_{0}^{2}-\mathrm{q}_{1}^{2}-\mathrm{q}_{2}^{2}\end{array}\right)\end{array}$ \\
\hline $\begin{array}{l}\text { Direction } \\
\text { Cosines }\end{array}$ & $\begin{array}{l}\delta \mathrm{C}_{\mathrm{b}}^{\mathrm{n}} \mathrm{f}^{\mathrm{b}}=\mathrm{F}_{\mathrm{VAC}}\left[\begin{array}{llllllllll}\delta \mathrm{c}_{11} & \delta \mathrm{c}_{12} & \delta \mathrm{c}_{13} & \delta \mathrm{c}_{21} & \delta \mathrm{c}_{22} & \delta \mathrm{c}_{23} & \delta \mathrm{c}_{31} & \delta \mathrm{c}_{32} & \delta \mathrm{c}_{33}\end{array}\right]^{\mathrm{T}} \\
\text { where: } \mathrm{F}_{\mathrm{VAC}}=\left(\begin{array}{cccccccccc}\mathrm{f}_{\mathrm{x}} & \mathrm{f}_{\mathrm{y}} & \mathrm{f}_{\mathrm{z}} & 0 & 0 & 0 & 0 & 0 & 0 \\
0 & 0 & 0 & \mathrm{f}_{\mathrm{x}} & \mathrm{f}_{\mathrm{y}} & \mathrm{f}_{\mathrm{z}} & 0 & 0 & 0 \\
0 & 0 & 0 & 0 & 0 & 0 & \mathrm{f}_{\mathrm{x}} & \mathrm{f}_{\mathrm{y}} & \mathrm{f}_{\mathrm{y}}\end{array}\right)\end{array}$ \\
\hline
\end{tabular}
table 1. and table 2.

Table 1 Calculation of term $\mathrm{C}_{\mathrm{b}}^{\mathrm{n}} \delta \mathrm{f}^{\mathrm{b}}$ 

Table (2) Calculation of term $\delta \mathrm{C}_{\mathrm{b}}^{\mathrm{n}} \mathrm{f}^{\mathrm{b}}$

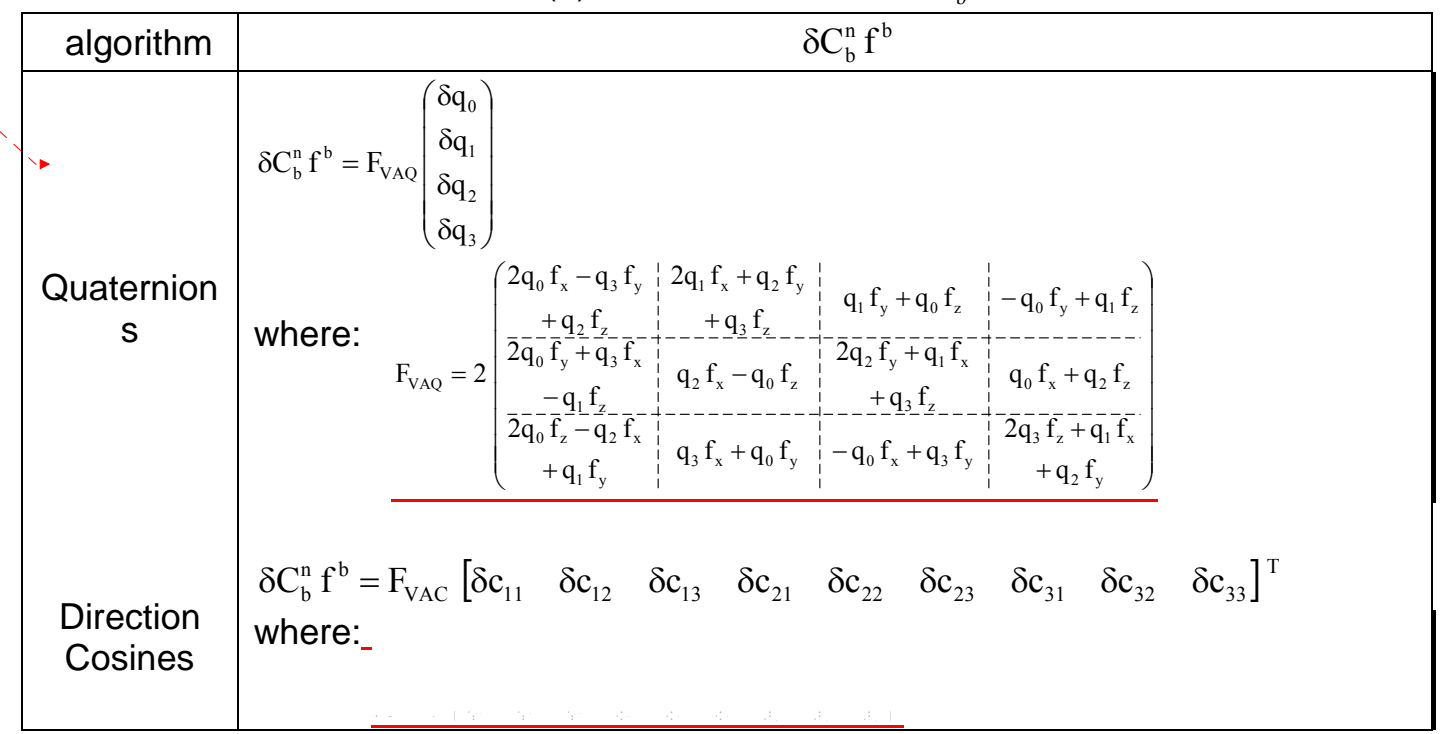

\section{4- ATTITUDE ERROR DYNAMICS}

The attitude of the vehicle relative to the $\mathrm{N}$-frame can be variety of set of variablt the most popular being Euler angles, direction cosines and quaternions.

\section{1- Attitude Error Dynamics Based on Quaternions}

We define the quaternions error as the arithmetic difference between the quaterni estimate, and the true quaternion.

$$
\delta \mathrm{q}_{\mathrm{n}}^{\mathrm{b}}=\overline{\mathrm{q}}_{\mathrm{n}}^{\mathrm{b}}-\mathrm{q}_{\mathrm{n}}^{\mathrm{b}}
$$

Attitude Error Dynamics can be expressed as [4] given in equation (11).

$$
\delta \dot{\mathrm{q}}_{\mathrm{n}}^{\mathrm{b}}=0.5 \Omega_{\mathrm{ib}}^{\mathrm{b}} \delta \mathrm{q}_{\mathrm{n}}^{\mathrm{b}}+0.5 \delta \Omega_{\mathrm{ib}}^{\mathrm{b}} \mathrm{q}_{\mathrm{n}}^{\mathrm{b}}
$$

where:

$$
\begin{gathered}
\frac{1}{2} \Omega_{\mathrm{ib}}^{\mathrm{b}} \delta \mathrm{q}_{\mathrm{n}}^{\mathrm{b}}=\mathrm{F}_{\mathrm{AAQ}}\left(\begin{array}{l}
\delta \mathrm{q}_{0} \\
\delta \mathrm{q}_{1} \\
\delta \mathrm{q}_{2} \\
\delta \mathrm{q}_{3}
\end{array}\right) \\
\mathrm{F}_{\mathrm{AAQ}}=\left(\begin{array}{cccc}
0 & -0.5 \omega_{\mathrm{x}} & -0.5 \omega_{\mathrm{y}} & -0.5 \omega_{\mathrm{z}} \\
0.5 \omega_{\mathrm{x}} & 0 & 0.5 \omega_{\mathrm{z}} & -0.5 \omega_{\mathrm{y}} \\
0.5 \omega_{\mathrm{y}} & -0.5 \omega_{\mathrm{z}} & 0 & 0.5 \omega_{\mathrm{x}} \\
0.5 \omega_{\mathrm{z}} & 0.5 \omega_{\mathrm{y}} & -0.5 \omega_{\mathrm{x}} & 0
\end{array}\right)
\end{gathered}
$$




$$
\mathrm{G}_{\mathrm{AAQ}}=\frac{1}{2}\left(\begin{array}{rrr}
-\overline{\mathrm{q}}_{1} & -\overline{\mathrm{q}}_{2} & -\overline{\mathrm{q}}_{3} \\
\overline{\mathrm{q}}_{0} & -\overline{\mathrm{q}}_{3} & \overline{\mathrm{q}}_{2} \\
\overline{\mathrm{q}}_{3} & \overline{\mathrm{q}}_{0} & -\overline{\mathrm{q}}_{1} \\
-\overline{\mathrm{q}}_{2} & \overline{\mathrm{q}}_{1} & \overline{\mathrm{q}}_{0}
\end{array}\right) \quad \frac{\frac{1}{2} \delta \Omega_{\mathrm{ib}}^{\mathrm{b}} \mathrm{q}_{\mathrm{n}}^{\mathrm{b}}=\mathrm{G}_{\mathrm{AAQ}}\left(\begin{array}{c}
\delta \omega_{\mathrm{x}} \\
\delta \omega_{\mathrm{x}} \\
\delta \omega_{\mathrm{x}}
\end{array}\right)}{}
$$

\section{2- Attitude Error Dynamics Based on Direction Cosines}

The attitude dynamics based on direction cosines is expressed by:

$$
\dot{\mathrm{r}}^{\mathrm{n}}=\dot{\mathrm{C}}_{\mathrm{b}}^{\mathrm{n}} \mathrm{r}^{\mathrm{b}}
$$

Where: $\mathrm{C}_{\mathrm{b}}^{\mathrm{n}}$ is the direction cosine matrix (DCM), which represents the transformati $\mathrm{B}$-frame to $\mathrm{N}$-frame, $\mathrm{r}^{\mathrm{n}}$ is the position vector in $\mathrm{N}$-frame and $\mathrm{r}^{\mathrm{b}}$ is the position vec in B-frame as shown in the following figure.

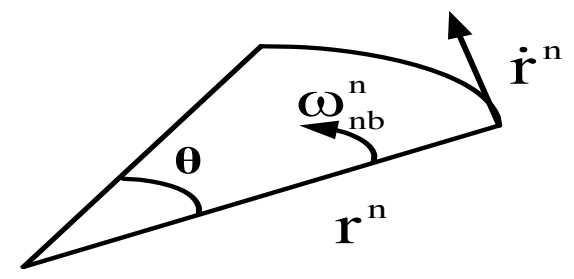

The velocity vector in $\mathrm{N}$-frame can be expressed as given in equation (15) [5].

$$
\begin{aligned}
\dot{\mathrm{r}}^{\mathrm{n}} & =\omega_{\mathrm{nb}}^{\mathrm{n}} \times \mathrm{r}^{\mathrm{n}} \\
& =\Omega_{\mathrm{nb}}^{\mathrm{n}} \mathrm{r}^{\mathrm{n}} \\
& =\mathrm{C}_{\mathrm{b}}^{\mathrm{n}} \Omega_{\mathrm{nb}}^{\mathrm{b}} \mathrm{r}^{\mathrm{b}}
\end{aligned}
$$

Where: $\omega_{\mathrm{nb}}^{\mathrm{n}}$ is the projection of the rotating rate vector of the B-frame with respect the $\mathrm{N}$-frame on the $\mathrm{N}$-frame and $\Omega_{\mathrm{nb}}^{\mathrm{n}}$ is the skew-symmetric matrix, corresponding $\omega_{\mathrm{nb}}^{\mathrm{n}}$.

Depending on the form that the derivative of DCM $\dot{C}_{b}^{n}$ can be expressed, $t$ methods can be extracted where $\dot{\mathrm{C}}_{\mathrm{b}}^{\mathrm{n}}=\mathrm{C}_{\mathrm{b}}^{\mathrm{n}} \Omega_{\mathrm{nb}}^{\mathrm{b}}$ represents method one $\mathrm{a}$ $\dot{\mathrm{C}}_{\mathrm{b}}^{\mathrm{n}}=-\Omega_{\mathrm{bn}}^{\mathrm{n}} \mathrm{C}_{\mathrm{b}}^{\mathrm{n}}$ represents method two. 


\section{5- IMPLEMENTATION OF THE INS/GPS KALMAN FILTER}

The INS/GPS Kalman Filter implementation is divided into four steps, as explained following subsections.

\section{1- Continuous System Equations}

$$
\dot{\chi}=\mathrm{F} \chi+\mathrm{Gu}
$$

Where: $\mathrm{F}$ is the dynamics matrix (state matrix), $\chi$ is the state vector, $\mathrm{u}$ is the forci vector function (input vector), and $G$ is a design matrix (input matrix). The terms are described jin details by the following subsection.

\section{- 5.1.1- Error of Position, Velocity, and Attitude Based on Quaternions}

Continuous system equations of error of position, velocity and attitude based quaternions can be expressed as follows:

$$
\begin{aligned}
& \chi=\left(\begin{array}{c}
\delta \mathrm{r}^{\mathrm{n}} \\
\delta \mathrm{v}^{\mathrm{n}} \\
\delta \mathrm{q}_{\mathrm{b}}^{\mathrm{n}}
\end{array}\right)=\left[\begin{array}{llllllllll}
\delta \varphi & \delta \lambda & \delta \mathrm{h} & \delta \mathrm{v}_{\mathrm{N}} & \delta \mathrm{v}_{\mathrm{E}} & \delta \mathrm{v}_{\mathrm{D}} & \delta \mathrm{q}_{0} & \delta \mathrm{q}_{1} & \delta \mathrm{q}_{2} & \delta \mathrm{q}_{3}
\end{array}\right]^{\mathrm{T}} \\
& \mathrm{u}=\left(\begin{array}{c}
\delta \mathrm{f}^{\mathrm{b}} \\
\delta \omega_{\mathrm{ib}}^{\mathrm{b}}
\end{array}\right)=\left[\begin{array}{llllll}
\delta \mathrm{f}_{\mathrm{x}} & \delta \mathrm{f}_{\mathrm{y}} & \delta \mathrm{f}_{\mathrm{z}} & \delta \omega_{\mathrm{x}} & \delta \omega_{\mathrm{y}} & \delta \omega_{\mathrm{z}}
\end{array}\right]^{\mathrm{T}} \\
& \mathrm{F}=\left(\begin{array}{ccc}
\mathrm{F}_{\mathrm{RR}} & \mathrm{F}_{\mathrm{RV}} & 0_{3 \times 4} \\
\mathrm{~F}_{\mathrm{VR}} & \mathrm{F}_{\mathrm{VV}} & \mathrm{F}_{\mathrm{VAQ}} \\
0_{4 \times 4} & 0_{4 \times 4} & \mathrm{~F}_{\mathrm{AAQ}}
\end{array}\right){ }^{\prime} \mathrm{G}=\left(\begin{array}{cc}
0_{3 \times 3} & 0_{3 \times 3} \\
\mathrm{G}_{\mathrm{AVQ}} & 0_{3 \times 3} \\
0_{4 \times 3} & \mathrm{G}_{\mathrm{AAQ}}
\end{array}\right)
\end{aligned}
$$

The details of matrix $\mathrm{F}$ and matrix $\mathrm{G}$ are given in [5].

\section{-5.1.2- Error of Position, Velocity, and Attitude Based on Direction Cosin Method One}

Continuous system equations of error of position, velocity and attitude based direction cosines can be expressed as follows:

$$
\begin{aligned}
& \chi=\left(\begin{array}{c}
\delta \mathrm{r}^{\mathrm{n}} \\
\delta \mathrm{v}^{\mathrm{n}} \\
\delta \mathrm{C}_{\mathrm{b}}^{\mathrm{n}}
\end{array}\right)=\left[\begin{array}{cccccccccc}
\delta \varphi & \delta \lambda & \delta \mathrm{h} & \delta \mathrm{v}_{\mathrm{N}} & \delta \mathrm{v}_{\mathrm{E}} & \delta \mathrm{v}_{\mathrm{D}} & & & & \\
\delta \mathrm{c}_{11} & \delta \mathrm{c}_{12} & \delta \mathrm{c}_{13} & \delta \mathrm{c}_{21} & \delta \mathrm{c}_{22} & \delta \mathrm{c}_{23} & \delta \mathrm{c}_{31} & \delta \mathrm{c}_{32} & \delta \mathrm{c}_{33}
\end{array}\right]^{\mathrm{T}} \\
& \mathrm{u}=\left(\begin{array}{c}
\delta \mathrm{f}^{\mathrm{b}} \\
\delta \omega_{\mathrm{ib}}^{\mathrm{b}}
\end{array}\right)=\left[\begin{array}{llllll}
\delta f_{\mathrm{x}} & \delta \mathrm{f}_{\mathrm{y}} & \delta \mathrm{f}_{z} & \delta \omega_{\mathrm{x}} & \delta \omega_{\mathrm{y}} & \delta \omega_{\mathrm{z}}
\end{array}\right]^{\mathrm{T}} \\
& F=\left(\begin{array}{lll}
F_{R R} & F_{R V} & 0_{3 \times 9} \\
F_{V R} & F_{V V} & F_{V A C} \\
0_{9 \times 3} & 0_{9 \times 3} & F_{A A C l}
\end{array}\right) \perp G=\left(\begin{array}{cc}
0_{3 \times 3} & 0_{3 \times 3} \\
G_{A V C} & 0_{3 \times 3} \\
0_{9 \times 3} & G_{A A C l}
\end{array}\right)
\end{aligned}
$$

The details of matrix $F$ and matrix $G$ are given in [5]. 
pt I منسّق: (Latin) Style Justified Black Bold (Latin) ... :spacing Line

محذذوف: Continuous System Equations of

محذوف: Direction Cosines Using Alternative Algorithm

محذوف: , and

محذوف: direction cosines using alternative algorithm

محذوف: is

محذذوف: The

\section{-5.1.3- Error of Position, Velocity, and Attitude Based on Direction cosin} method one Method two

Continuous system equations of error of position, velocity and attitude based direction cosines method one matrix can be constructed by augmenting Eq. (1), ( and خطأ لم يتم العثور على مصدر المرجع. as follows:

$$
\begin{aligned}
& \chi=\left(\begin{array}{c}
\delta \mathrm{r}^{\mathrm{n}} \\
\delta \mathrm{v}^{\mathrm{n}} \\
\delta \mathrm{c}_{\mathrm{b}}^{\mathrm{n}}
\end{array}\right)=\left[\begin{array}{cccccccccc}
\delta \varphi & \delta \lambda & \delta \mathrm{h} & \delta \mathrm{v}_{\mathrm{N}} & \delta \mathrm{v}_{\mathrm{E}} & \delta \mathrm{v}_{\mathrm{D}} \\
\delta \mathrm{c}_{11} & \delta \mathrm{c}_{12} & \delta \mathrm{c}_{13} & \delta \mathrm{c}_{21} & \delta \mathrm{c}_{22} & \delta \mathrm{c}_{23} & \delta \mathrm{c}_{31} & \delta \mathrm{c}_{32} & \delta \mathrm{c}_{33}
\end{array}\right]^{\mathrm{T}} \\
& \mathrm{u}=\left(\begin{array}{c}
\delta \mathrm{f}^{\mathrm{b}} \\
\delta \omega_{\mathrm{ib}}^{\mathrm{b}}
\end{array}\right)=\left[\begin{array}{llllll}
\delta \mathrm{f}_{\mathrm{x}} & \delta \mathrm{f}_{\mathrm{y}} & \delta \mathrm{f}_{\mathrm{z}} & \delta \omega_{\mathrm{x}} & \delta \omega_{\mathrm{y}} & \delta \omega_{\mathrm{z}}
\end{array}\right]^{\mathrm{T}} \\
& F=\left(\begin{array}{lll}
F_{R R} & F_{R V} & 0_{3 \times 9} \\
F_{\mathrm{VR}} & F_{\mathrm{VV}} & F_{\mathrm{VAC}} \\
0_{9 \times 3} & 0_{9 \times 3} & \mathrm{~F}_{\mathrm{AAC} 2}
\end{array}\right)=\left(\begin{array}{cc}
0_{3 \times 3} & 0_{3 \times 3} \\
\mathrm{G}_{\mathrm{AVC}} & 0_{3 \times 3} \\
0_{9 \times 3} & \mathrm{G}_{\mathrm{AAC} 2}
\end{array}\right)
\end{aligned}
$$

The details of matrix $\mathrm{F}$ and matrix $\mathrm{G}$ are given in [5].

\section{2- CONVERT THE CONTINUOUS SYSTEM EQUATIONS TO DISCRE' EQUATIONS}

$$
\chi\left(\mathrm{t}_{\mathrm{k}+1}\right)=\phi\left(\mathrm{t}_{\mathrm{k}+1}, \mathrm{t}_{\mathrm{k}}\right) \chi\left(\mathrm{t}_{\mathrm{k}}\right)+\int_{\mathrm{t}_{\mathrm{k}}}^{\mathrm{t}_{\mathrm{k}+1}} \phi\left(\mathrm{t}_{\mathrm{k}+1}, \tau\right) \mathrm{G}(\tau) \mathrm{u}(\tau) \mathrm{d} \tau
$$

or in abbreviated notation

$$
\chi_{\mathrm{k}+1}=\Phi_{\mathrm{k}} \chi_{\mathrm{k}}+\mathrm{w}_{\mathrm{k}}
$$

where: $\chi_{\mathrm{k}}$ is the state vector at time $\mathrm{t}_{\mathrm{k}}, \phi_{\mathrm{k}}$ is the state transition matrix at time $\mathrm{w}_{\mathrm{k}}$ is the vector of process noise at time $\mathrm{t}_{\mathrm{k}}$.

The covariance matrix associated with $\mathrm{w}_{\mathrm{k}}$ is:

$$
\mathrm{E}\left[\mathrm{w}_{\mathrm{k}} \mathrm{w}_{\mathrm{i}}^{\mathrm{T}}\right]=\left\{\begin{array}{lc}
\mathrm{Q} & \mathrm{i}=\mathrm{k} \\
0 & \mathrm{i} \neq \mathrm{k}
\end{array}\right.
$$

where: $\mathrm{Q}$ is the covariance matrix of process noise in the system state.

The numerical method to find the state transition matrix over short time inter $\Delta \mathrm{t}=\mathrm{t}_{\mathrm{k}+1}-\mathrm{t}_{\mathrm{k}}$ is preferred:

$$
\Phi_{\mathrm{k}}=\exp (\mathrm{F} \Delta \mathrm{t}) \approx \mathrm{I}+\mathrm{F} \Delta \mathrm{t}
$$

The equation for calculating covariance matrix of process noise in the system state time $\mathrm{t}_{\mathrm{k}}\left(\mathrm{Q}_{\mathrm{k}}\right)$ is given by equation (30).

$$
\begin{aligned}
\mathrm{Q}_{\mathrm{K}} & =\mathrm{E}\left[\mathrm{w}_{\mathrm{K}} \mathrm{w}_{\mathrm{k}}^{\mathrm{T}}\right] \\
& =\mathrm{E}\left\{\left[\int_{t_{\mathrm{k}}}^{t_{k+1}} \phi\left(\mathrm{t}_{\mathrm{k}+1}, \xi\right) \mathrm{G}(\xi) \mathrm{u}(\xi) \mathrm{d} \xi\right]\left[\int_{t_{\mathrm{k}}}^{t_{k+1}} \phi\left(\mathrm{t}_{\mathrm{k}+1}, \xi\right) \mathrm{G}(\xi) \mathrm{u}(\xi) \mathrm{d} \xi\right]^{\mathrm{T}}\right\} \\
& =\int_{t_{\mathrm{k}}}^{t_{k+1}} \int_{t_{\mathrm{k}}}^{t_{k+1}} \phi\left(\mathrm{t}_{\mathrm{k}+1}, \xi\right) \mathrm{G}(\xi) \mathrm{E}\left[\mathrm{u}(\xi) \mathrm{u}^{\mathrm{T}}(\eta)\right] \mathrm{G}^{\mathrm{T}}(\eta) \phi^{\mathrm{T}}\left(\mathrm{t}_{\mathrm{k}+1}, \eta\right) \mathrm{d} \xi \mathrm{d} \eta
\end{aligned}
$$


محذوف: This estimated does not account for any of the correlations between the components of the driving $\mathrm{W}$ that develop over the course of a sampling period because of the

integration of the continuoustime driving noise through the state dynamics Therefore, in this research

محذوف: estimated
The $Q_{k}$ is calculated using the first order estimation of the transition matrix, [5] expressed by equation (31).

$$
\mathrm{Q}_{\mathrm{k}} \approx \phi_{\mathrm{k}} \mathrm{GQG}{ }^{\mathrm{T}} \phi_{\mathrm{k}}^{\mathrm{T}} \Delta \mathrm{t}
$$

\section{3- OBSERVATION EQUATIONS}

The following observation equations

$$
z_{k}=H_{\mathrm{K}} \chi_{k}+v_{k}
$$

where: $z_{k}$ is the vector of measurement at time $t_{k}, \chi_{k}$ is the state vector at time $\mathrm{H}_{\mathrm{k}}$ is the measurement matrix at time $t_{k}$, and $v_{k}$ is the vector measurement noise at time $t_{k}$.

The covariance matrices for the $v_{k}$ is given by

$$
E\left[v_{k} v_{i}^{T}\right]=\left\{\begin{array}{cc}
R_{k} & i=k \\
0 & i \neq k
\end{array}\right.
$$

where: $R_{k}$ is the covariance matrix of noise measurement at time $t_{k}$.

The position and velocity from GPS can be considered as measurements. T straightforward formulation of the Observation equation can be written as:

$$
z_{\mathrm{K}}=\left(\begin{array}{c}
\mathrm{r}_{\mathrm{INS}}^{\mathrm{n}}-\mathrm{r}_{\mathrm{GPS}}^{\mathrm{n}} \\
-\mathrm{v}_{\mathrm{INS}}^{\mathrm{n}}--\mathrm{v}_{\mathrm{GPS}}^{\mathrm{n}}
\end{array}\right)=\left(\begin{array}{c}
\varphi_{\mathrm{INS}}-\varphi_{\mathrm{GPS}} \\
\lambda_{\mathrm{INS}}-\lambda_{\mathrm{GPS}} \\
\mathrm{h}_{\mathrm{INS}}-\mathrm{h}_{\mathrm{GPS}} \\
---------
\end{array}\right) \quad \mathrm{H}_{\mathrm{k}}=\left(\begin{array}{ccc}
\mathrm{I}_{3 \times 3} & 0_{3 \times 3} & 0_{3 \times 3} \\
0_{3 \times 3} & \mathrm{I}_{3 \times 3} & 0_{3 \times 3}
\end{array}\right)
$$

However, this approach causes numerical instabilities in calculati $\left[\mathrm{H}_{\mathrm{k}} \mathrm{P}_{\mathrm{k}}^{-} \mathrm{H}_{\mathrm{k}}^{\mathrm{T}}+\mathrm{R}_{\mathrm{k}}\right\rfloor^{-1}$ for the Kalman gain $\mathrm{K}_{\mathrm{k}}$. Because $\left(\varphi_{\mathrm{INS}}-\varphi_{\mathrm{GPS}}\right)$ and $\left(\lambda_{\mathrm{INS}}-\lambda_{\mathrm{Gl}}\right.$ are in radians and therefore they are very small values. This problem can resolved if the first and second rows are multiplied by $(\mathrm{M}+\mathrm{h})$ and $((\mathrm{N}+\mathrm{h}) \cos (\varphi$ respectively [5]. Hence, the Observation equation will take the form:

$$
\begin{aligned}
& z_{\mathrm{k}}=\left(\begin{array}{c}
(\mathrm{M}+\mathrm{h})\left(\varphi_{\mathrm{INS}}-\varphi_{\mathrm{GPS}}\right) \\
{[(\mathrm{N}+\mathrm{h}) \cos (\varphi)]\left(\lambda_{\mathrm{INS}}-\lambda_{\mathrm{GPS}}\right)} \\
\left(\mathrm{h}_{\mathrm{INS}}-\mathrm{h}_{\mathrm{GPS}}\right) \\
--------- \\
\mathrm{v}_{\mathrm{INS}}^{\mathrm{n}}-\mathrm{v}_{\mathrm{GPS}}^{\mathrm{n}}
\end{array}\right) \\
& \mathrm{H}_{\mathrm{k}}=\left(\begin{array}{ccc:c:c}
\mathrm{M}+\mathrm{h} & 0 & 0 & & \\
0 & (\mathrm{~N}+\mathrm{h}) \cos (\varphi) & 0 & 0_{3 \times 3} & 0_{3 \times 3} \\
0 & 0 & 1 & - & \\
\hdashline-0.3 \times 3 & & \mathrm{I}_{3 \times 3} & 0_{3 \times 3}
\end{array}\right)
\end{aligned}
$$

and the following is covariance matrix of noise measurement : 


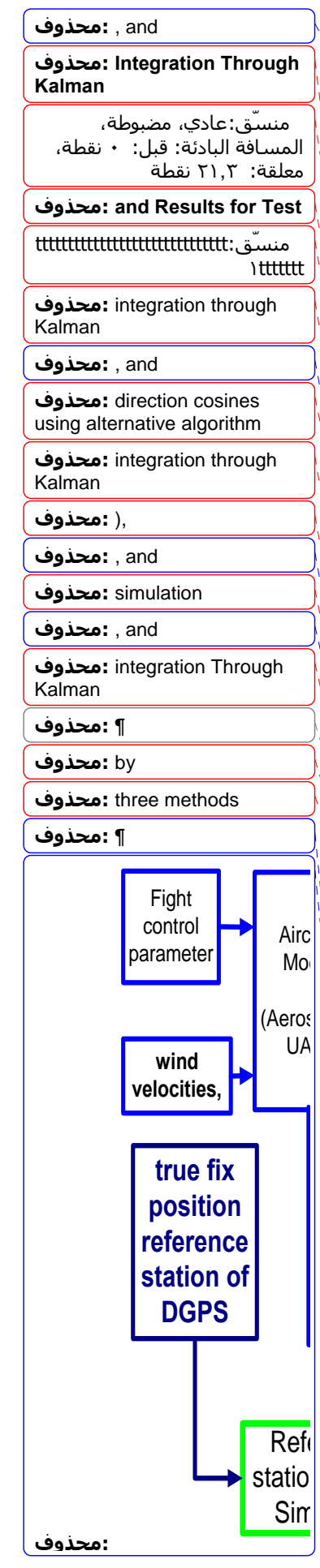

$$
\mathrm{R}_{\mathrm{k}}=\operatorname{diag}\left(\sigma_{\varphi}^{2} \sigma_{\lambda}^{2} \sigma_{\mathrm{h}}^{2} \sigma_{\mathrm{V}_{\mathrm{N}}}^{2} \sigma_{\mathrm{V}_{\mathrm{E}}}^{2} \sigma_{\mathrm{V}_{\mathrm{D}}}^{2}\right)
$$

which can be obtained from GPS processing.

\section{4- Kalman Filter Algorithm}

The Kalman filter can be divided into two stages, the update, and prediction. In $\mathrm{t}$ former, the Kalman gain, $\mathrm{K}_{\mathrm{k}}$ is computed first, and then the state and the er covariance are updated using the prior estimate, $\hat{\chi}_{\mathrm{k}}^{-}$and its error covariance, $\mathrm{P}_{\mathrm{k}}^{-}$:

in the prediction stage.

$$
\begin{aligned}
& \mathrm{K}_{\mathrm{k}}=\mathrm{P}_{\mathrm{k}}^{-} \mathrm{H}_{\mathrm{k}}^{\mathrm{T}}\left[\mathrm{H}_{\mathrm{k}} \mathrm{P}_{\mathrm{k}}^{-} \mathrm{H}_{\mathrm{k}}^{\mathrm{T}}+\mathrm{R}_{\mathrm{k}}\right]^{-1} \\
& \hat{\chi}_{\mathrm{k}}=\hat{\chi}_{\mathrm{k}}^{-}+\mathrm{K}_{\mathrm{k}}\left[\mathrm{z}_{\mathrm{k}}-\mathrm{H}_{\mathrm{k}} \hat{\chi}_{\mathrm{k}}^{-}\right] \\
& \mathrm{P}_{\mathrm{k}}=\left[\begin{array}{lll}
\mathrm{I}-\mathrm{K}_{\mathrm{k}} & \mathrm{H}_{\mathrm{k}}
\end{array}\right] \mathrm{P}_{\mathrm{k}}^{-}
\end{aligned}
$$

$$
\begin{gathered}
\hat{\chi}_{\mathrm{k}+1}^{-}=\Phi_{\mathrm{k}} \hat{\chi}_{\mathrm{k}} \\
\mathrm{P}_{\mathrm{k}+1}^{-}=\Phi_{\mathrm{k}} \mathrm{P}_{\mathrm{k}} \Phi_{\mathrm{k}}^{\mathrm{T}}+\mathrm{Q}_{\mathrm{k}}
\end{gathered}
$$

\section{6- SIMULATION OF GPS/INS INTEGRATION USING KALMAN FILTI ALGORITHMS}

Simulation for test algorithms of GPS/INS Integration using Kalman filter algorithı based on attitude by quaternions, direction cosines, and direction cosines meth one results will describe in following subsection.

\section{1- Block Diagram}

Chart of simulation block diagram for GPS/INS Jntegration using Kalman fil algorithms is shown in Fig.). It contains flight control parameters (flap, elevat aileron, rudder, throttle, mixture and ignition), The INS algorithm, The DGPS mod and the GPS/INS Integration module using Kalman filter.

The INS algorithm is one of strapdown INS algorithms for attitude calculation. It $c$ be calculated by either quaternions method [INSQ], direction cosines method o [INSC1].

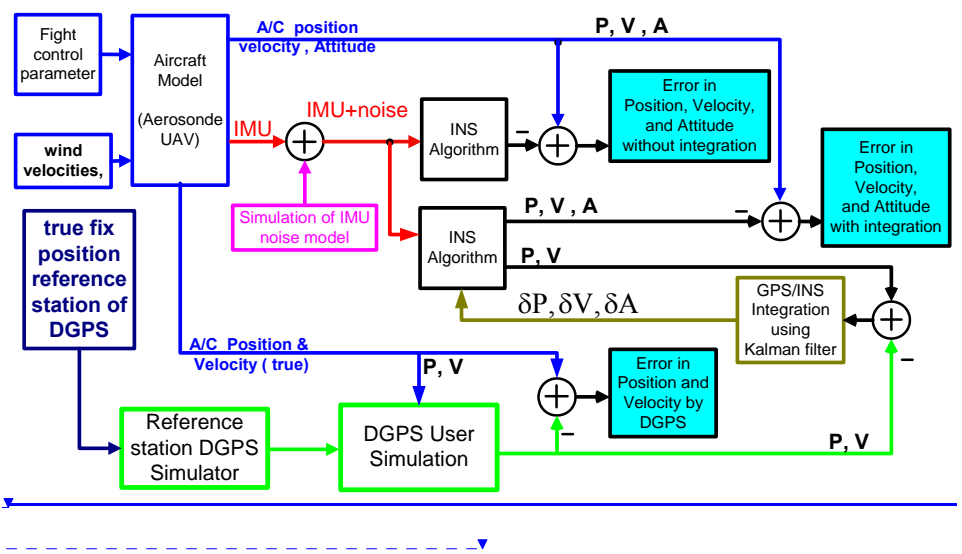




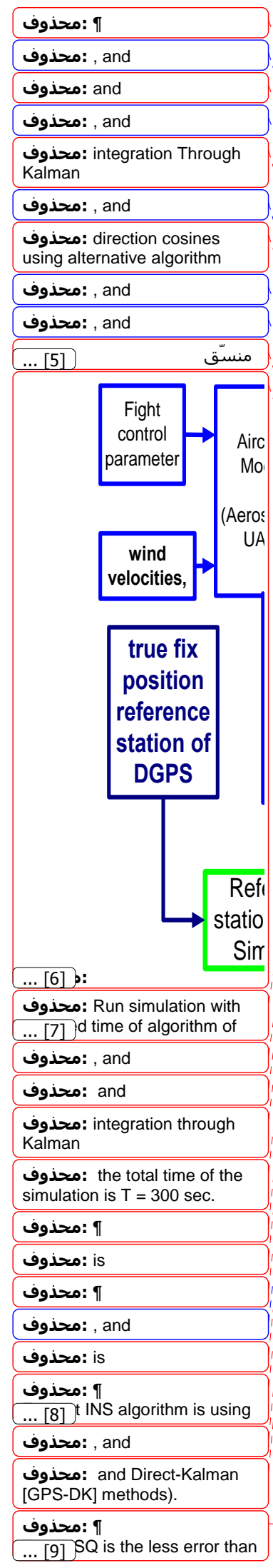

\section{Fig.2. Simulation block diagram for GPS/INS integration using Kalman}

,The DGPS simulation are dividing into two main parts, the first is the referen station, the second is the DGPS receiver, the calculation in receiver is done by thr methods Direct method [GPS-D], Kalman method [GPS-K] or Direct-Kalman meth [GPS-DK] . The difference between position, and velocity of outputs from DGPS a INS algorithms fed to the algorithm GPS/INS integration.

The GPS/INS Integration using Kalman filter in agreement with type of attitu calculation used for INS algorithms by three methods quaternions [INSQ], directi cosines method one [INSC1] and, direction cosines method two [INSC2].

The difference between position, velocity and attitude of aircraft model (true) a output from INS algorithm is the error in position, velocity and attitude as shown Fig.).

\section{-6.2- SIMULATION RESULT}

The INS data rate is taken every $0.01 \mathrm{sec}$, while the DGPS data is taken every $1 \mathrm{st}$ The sampled time of INS/GPS Integration using Kalman filter Ts (GPS/INS) $=1 \mathrm{sec}$ The position and velocity plots from aircraft model (Aerosonde UAV) for testi GPS/INS integration algorithms is considered as the true position and velocity shown in Fig.).

,The attitude plots from aircraft model (Aerosonde UAV) is considered as the tr attitude (roll $[\phi]$, pitch $[\theta]_{,}$and yaw $[\psi]$ ) are shown in Fig.).

.The error in position, velocity, and attitude_based on previous assumptions usi different methods of solution (Direct [GPS-D], Kalman [GPS-K], or Direct-Kalm [GPS-DK] methods) are shown in the Fig. 5, Fig. 6, Fig. 7, Fig. 8, Fig. 9, and Fig. 11
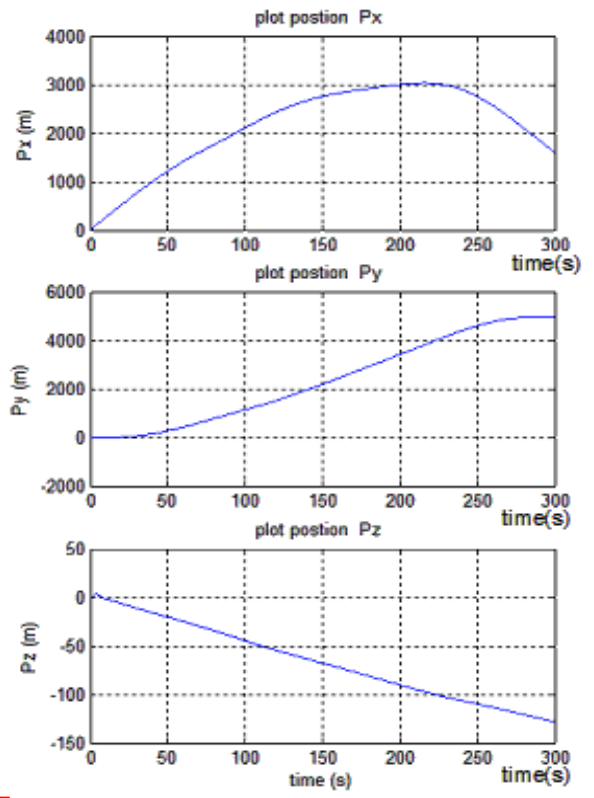
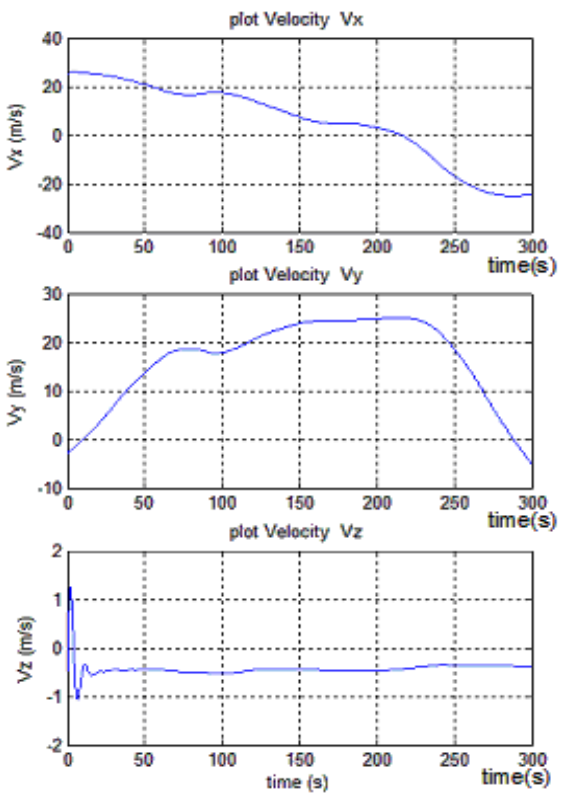
محذوف: integration through Kalman

\begin{tabular}{|c|}
\hline $\begin{array}{l}\text { محذوف: integration through } \\
\text { Kalman }\end{array}$ \\
\hline 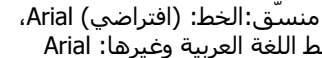 \\
\hline
\end{tabular}

Fig.1. Position and velocity of Aerosonde UAV for test INS/GPS Integration usinc Kalman filter
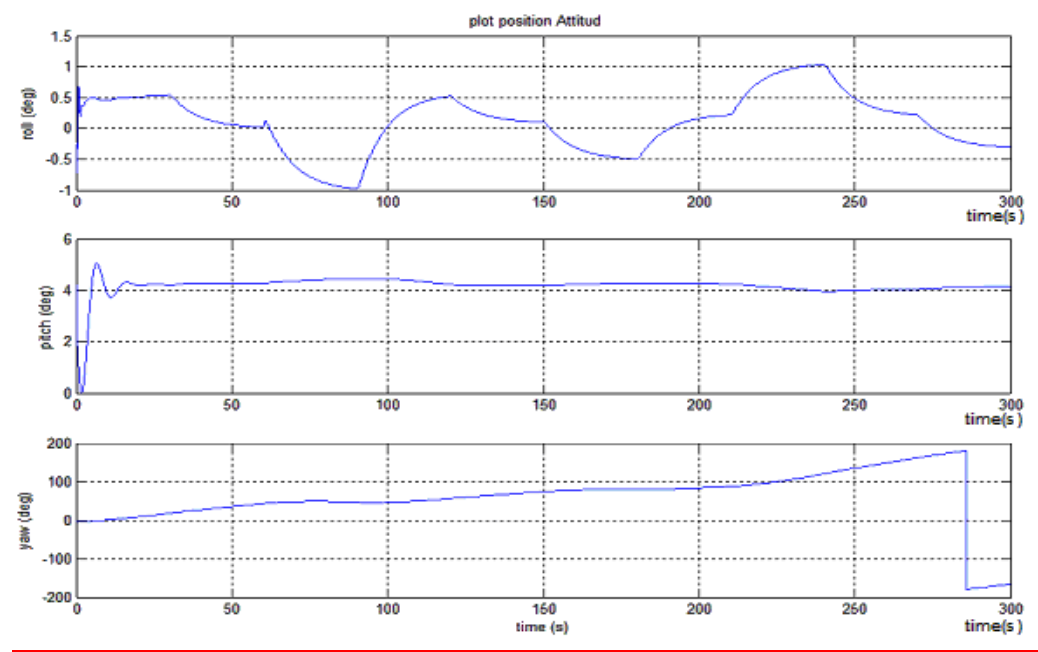

Fig.2. Attitude of Aerosonde UAV for test INS/GPS \ntegration using Kalman filte 

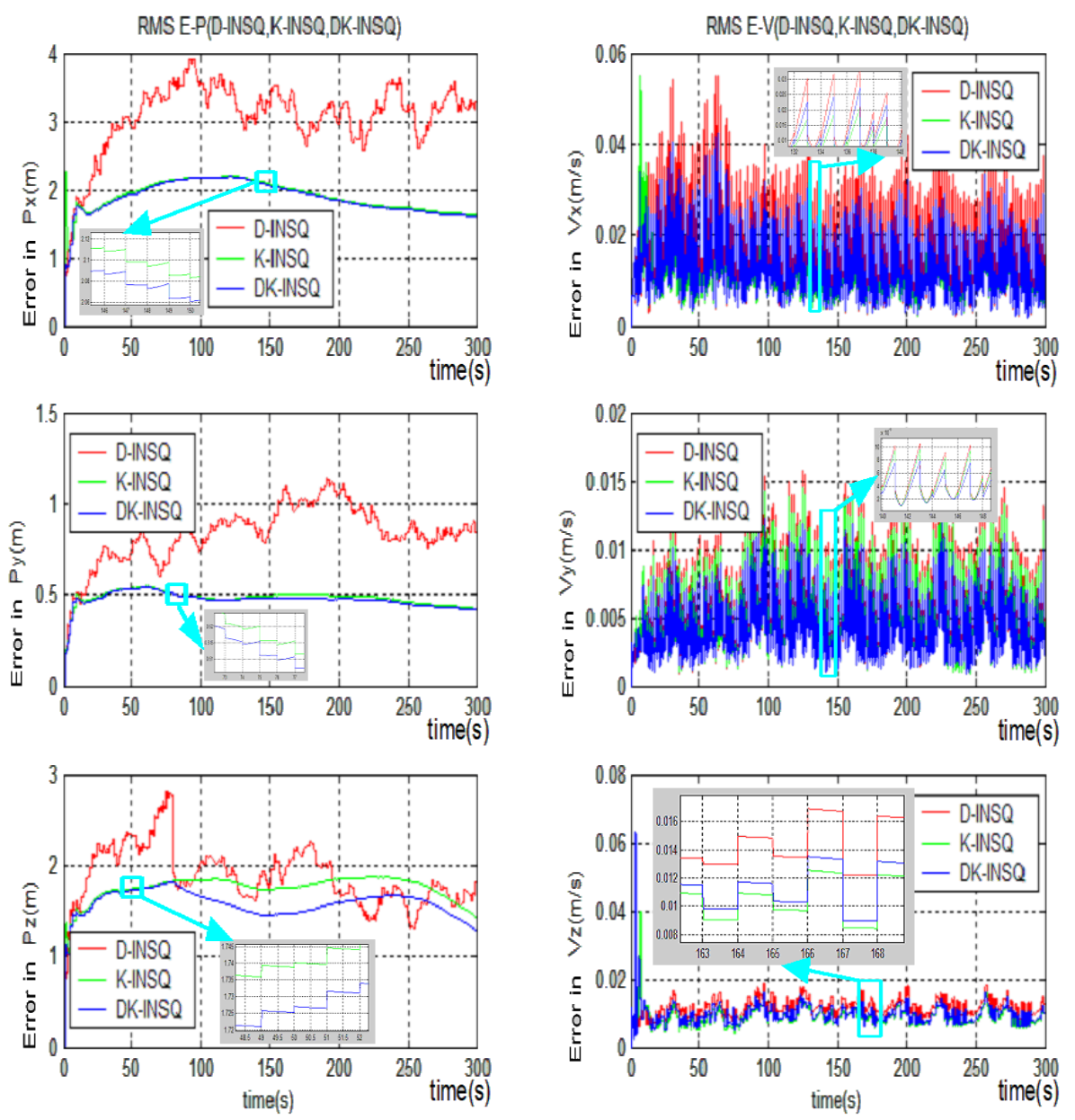

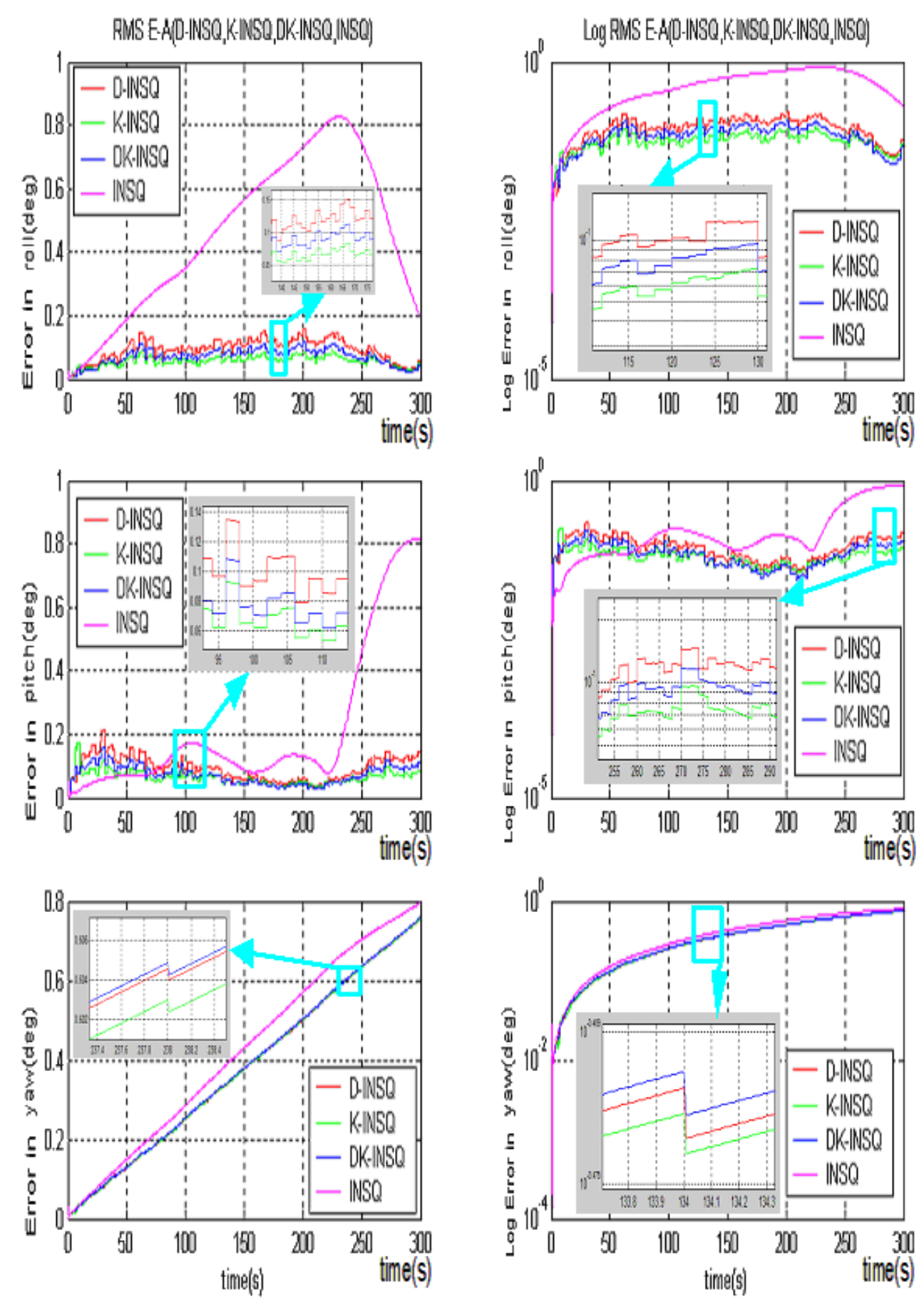

Fig.4. The RMS and Log RMS error in attitude of INS algorithm using quaternions 
محذوف: The INSC1 is the less error than anther algorithm in roll $(\phi)$ less error until 2.0687 sec from start, pitch $(\theta)$ less error until $80.0016 \mathrm{sec}$ from start, yaw $(\psi)$ less error until $34.0023 \mathrm{sec}$ from start.
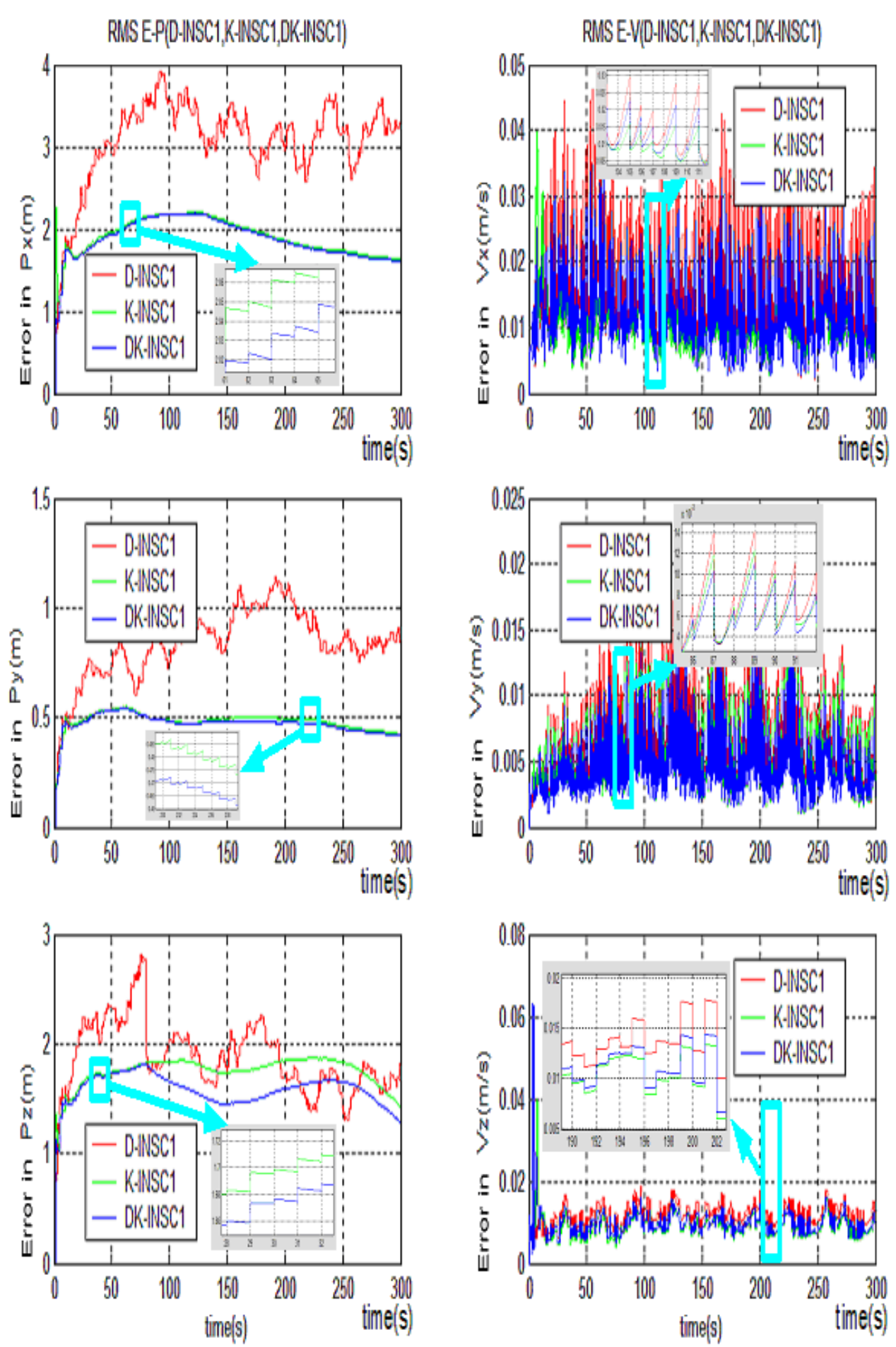

Fig.5. The RMS error in position and velocity of INS algorithm using direction cosines method one 

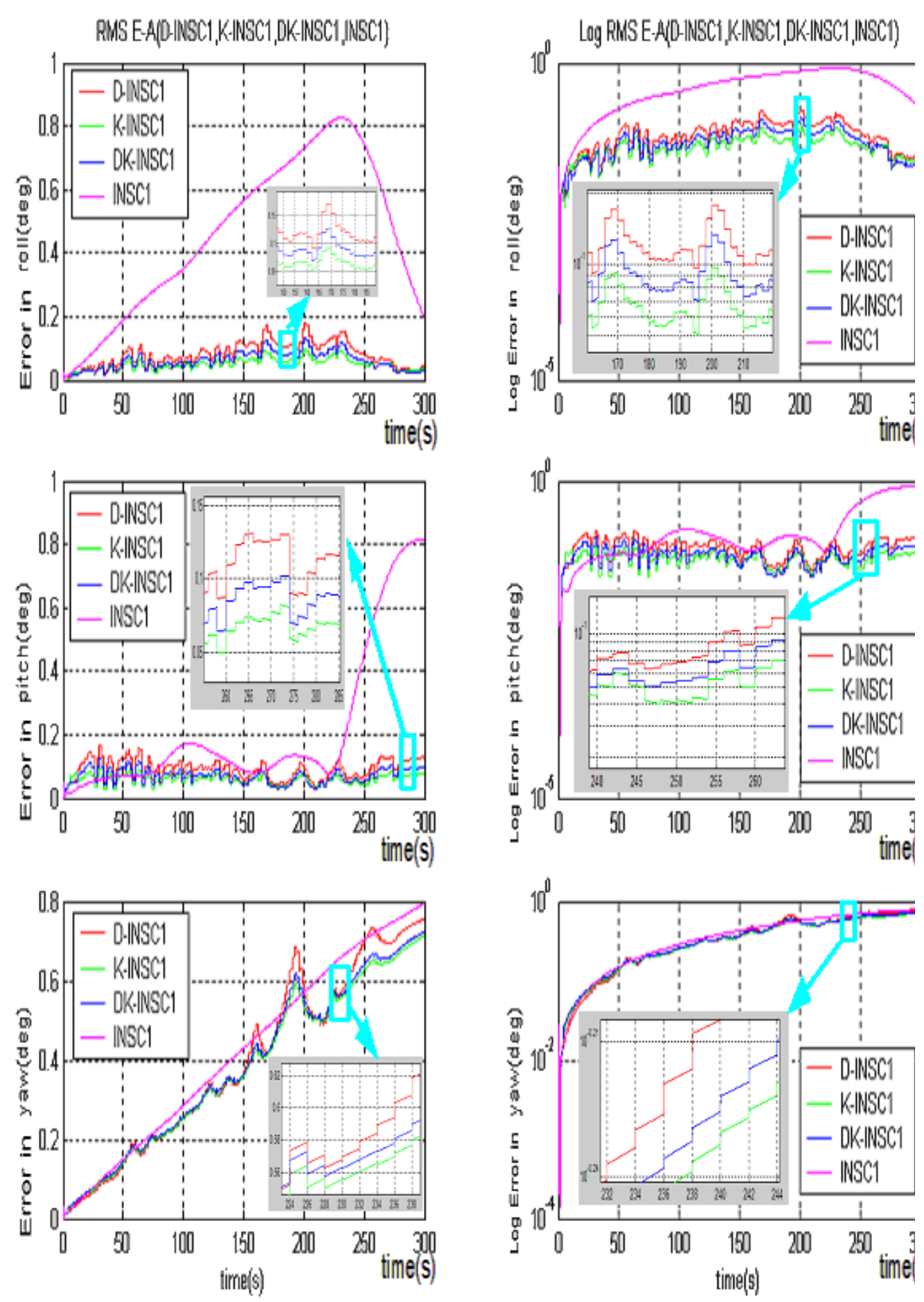

Fig.6. The RMS and Log RMS error in attitude of INS algorithm using direction cosines method one 

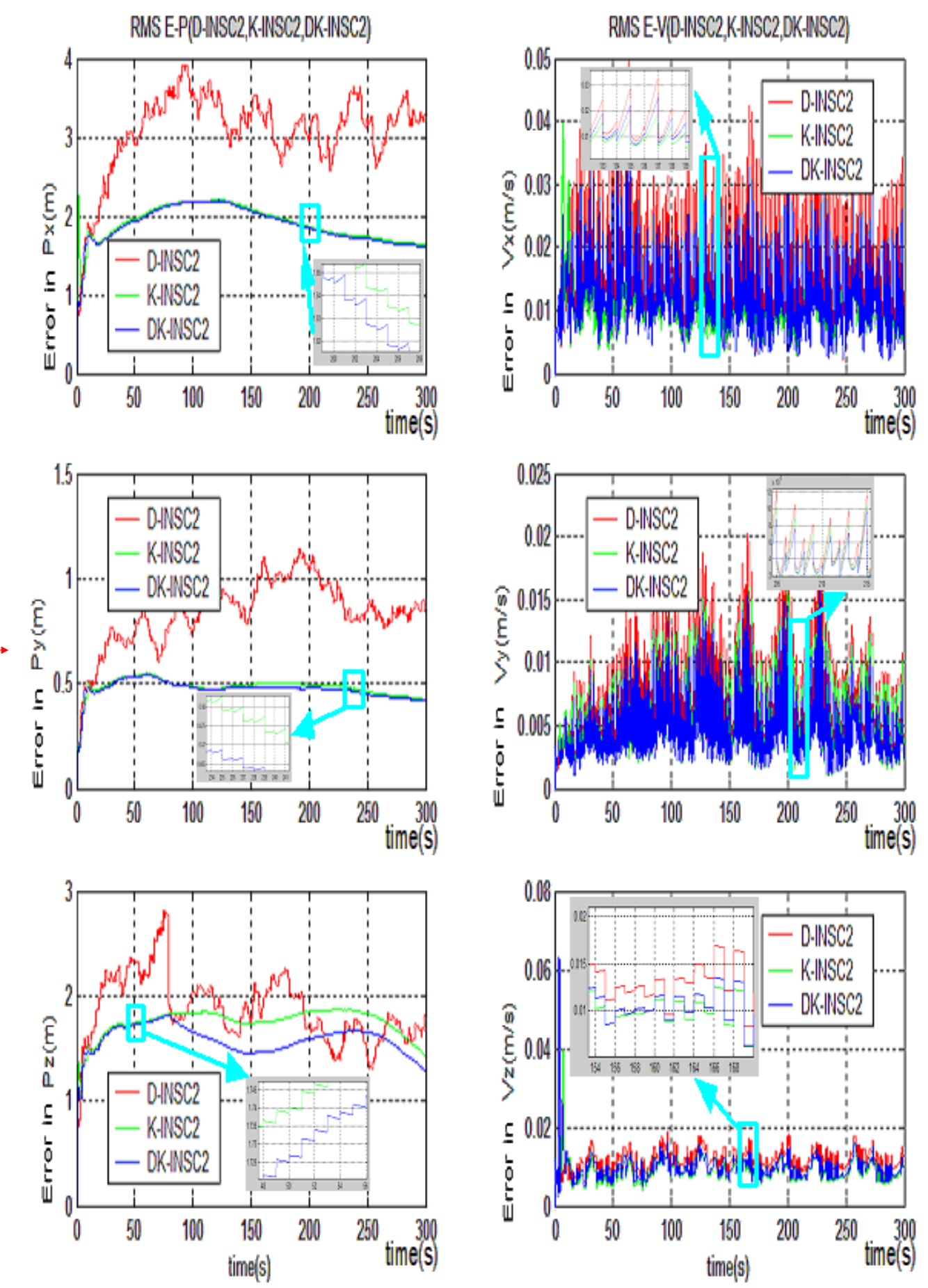

Fig.7. The RMS error in position and velocity of INS algorithm using direction cosin منسّق:متوسط method two 

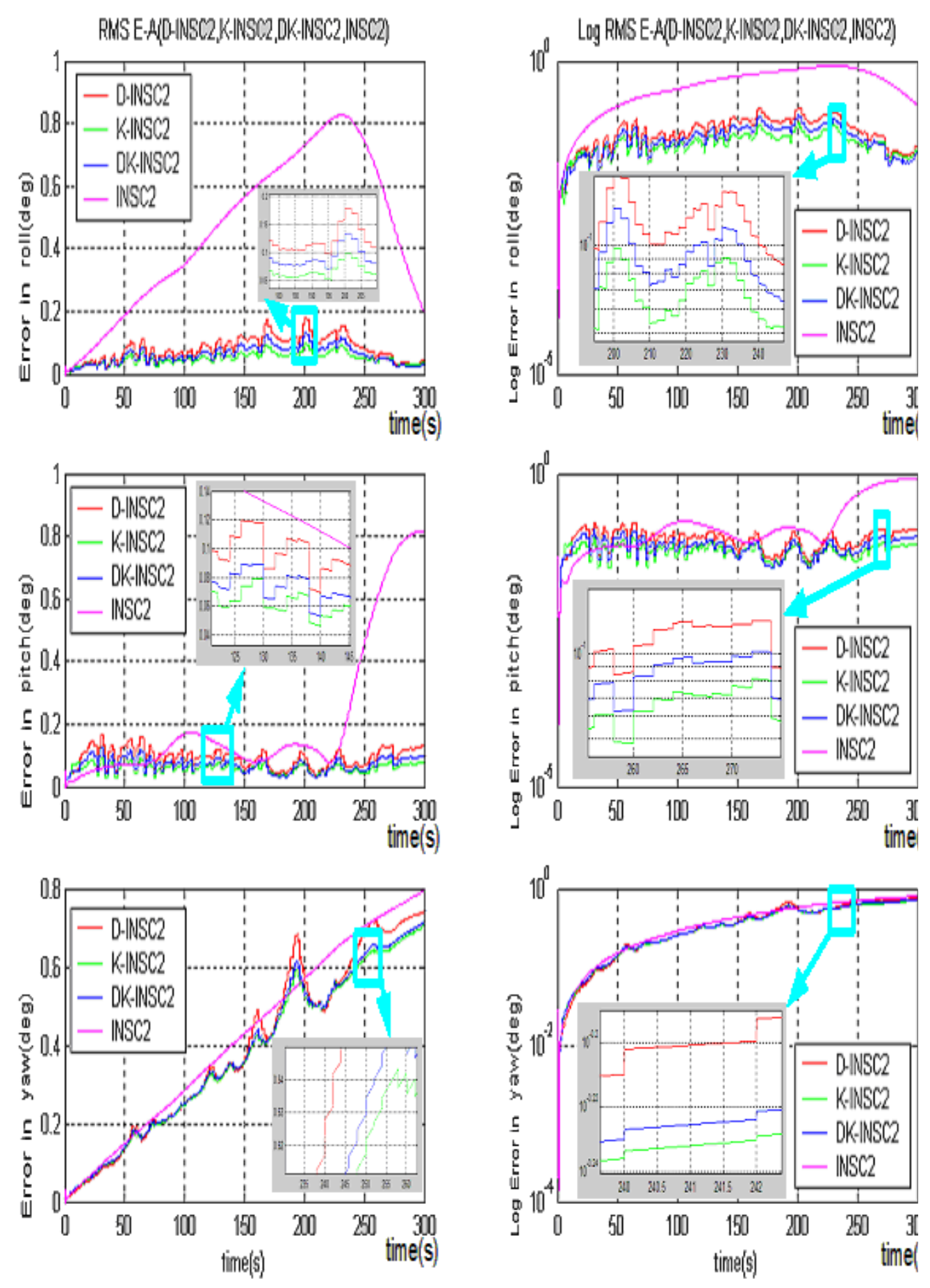

Fig.8. The RMS and Log RMS error in attitude of INS algorithm using direction cosines method two 
محذوف: The lowest RMS erro in position is INS algorithm is using quaternions for attitude integration with GPS using

Direct-Kalman to solve

navigation equation. ๆ

The lowest RMS error in velocity and attitude is INS algorithm is using direction cosines for attitude integration with GPS using Kalman filter to solve navigation equation. I

\section{CONCLUSION}

As GPS / INS integration methods have become increasingly sophisticated, it is oft no longer possible to test the sub-systems individually and extrapolate the combin performance from the separate results; they must be tested together. Evaluating $t$ performance of an integrated INS/GPS system requires the stimulation of both $t$ GPS and inertial sub-systems simultaneously. In this paper, simulation of differe GPS/INS integration methods is applied. The GPS motion data is used in a tim manner. In the implementation used, the simulated IMU data is subjected to an er model, which adds representative errors to the IMU. The simulation results explc that the integration of GPS using Direct-Kalman and JNS algorithm based quaternions is the most reliable algorithm.

\section{REFRENCES}

[1] AeroSim aeronautical simulation blockset Version 1.1, http://www.u dynamics.com.

[2] Godha, S. "Performance Evaluation of Low Cost MEMS-Based IMU Integrated With GPS for Land Vehicle Navigation Application", MSc Thesis, Department of Geomatics Engineering, The University of Calgary, Canada, (2006).

[3]Petovello, M.G. "Real-Time Integration of a Tactical-Grade IMU and GPS for Hig Accuracy Positioning and Navigation", Ph.D. Thesis, Department of Geomatics Engineering, The University of Calgary, Canada, (2003).

[4] Jau-Hsiun Wang" Intelligent MEMS INS/GPS Integration for Land Vehicle Navigation", Ph.D. Thesis, Department of Geomatics Engineering, The Universit of Calgary, Canada, (2006).

[5] Ahmed Azouz, " Integration Of GPS/INS Systems ", Msc. Thesis, MTC of Cairo, (2007).

[6] Brown G.R., "Introduction to Random Signal Analysis And applied Kalman Filtering", John Wiley \& Sons (1998).

[7] Eun-Hwan Shin, "Accuracy Improvement of Low Cost INS/GPS for Land Applications", Msc. Thesis, The University of Calgary, Alberta, December, (2001 
[1] Godha, S. "Performance Evaluation of Low Cost MEMS-Based IMU Integra1 With GPS for Land Vehicle Navigation Application", MSc Thesis, Department Geomatics Engineering, The University of Calgary, Canada, (2006).

[2] Petovello, M.G. "Real-Time Integration of a Tactical-Grade IMU and GPS High-Accuracy Positioning and Navigation", Ph.D. Thesis, Department of Geomat Engineering, The University of Calgary, Canada, (2003).

[3] Jau-Hsiun Wang" Intelligent MEMS INS/GPS Integration for Land Vehi Navigation", Ph.D. Thesis, Department of Geomatics Engineering, The University Calgary, Canada, (2006).

[4] Ahmed Azouz, " Integration Of GPS/INS Systems ", Msc. Thesis, MTC of Cairo, (2007

[5] Eun-Hwan Shin, "Accuracy Improvement of Low Cost INS/GPS for Land Application Msc. Thesis, The University of Calgary, Alberta, December, (2001). 


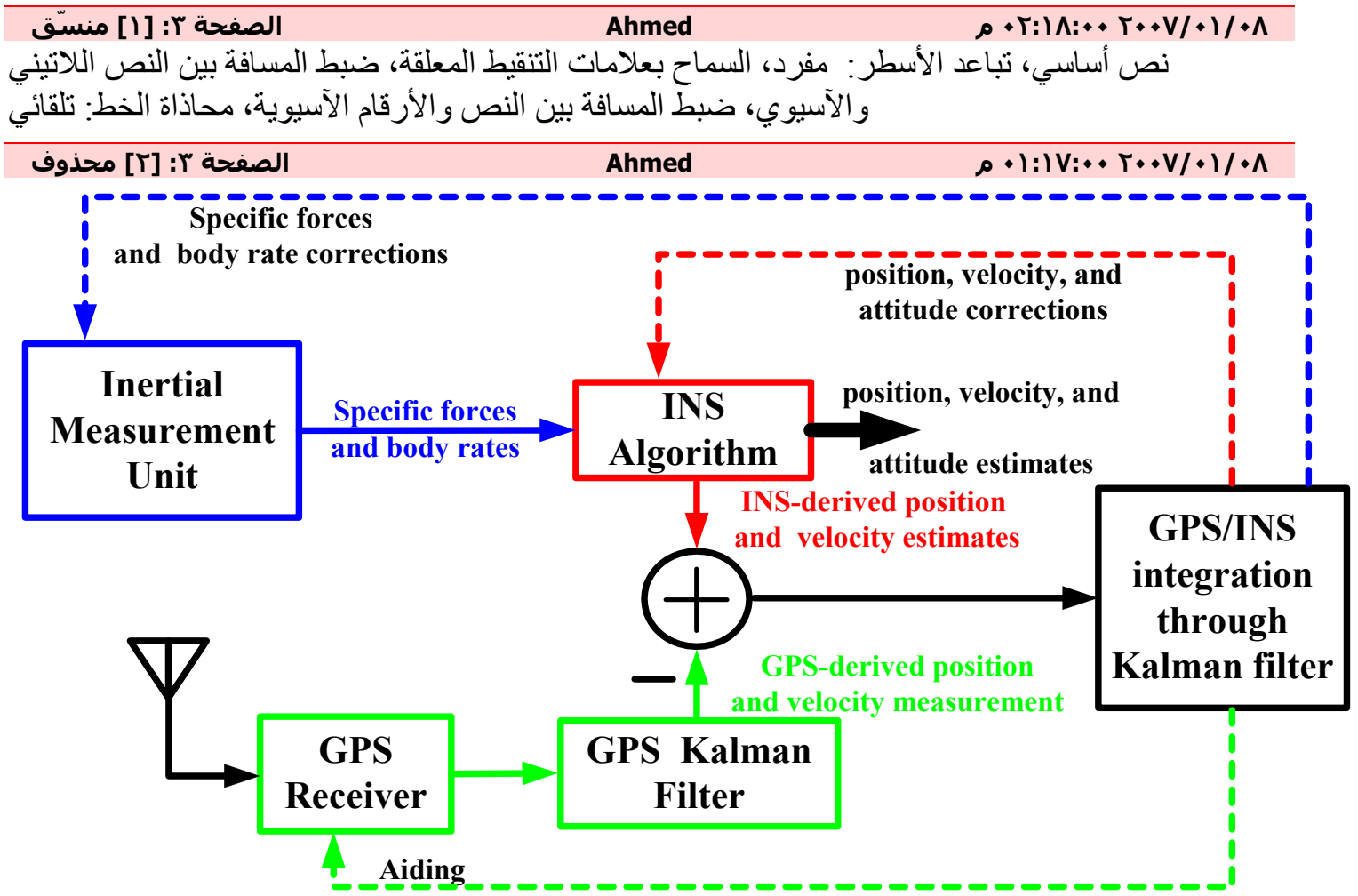

Fig(4.1): GPS/INS system with loose integration

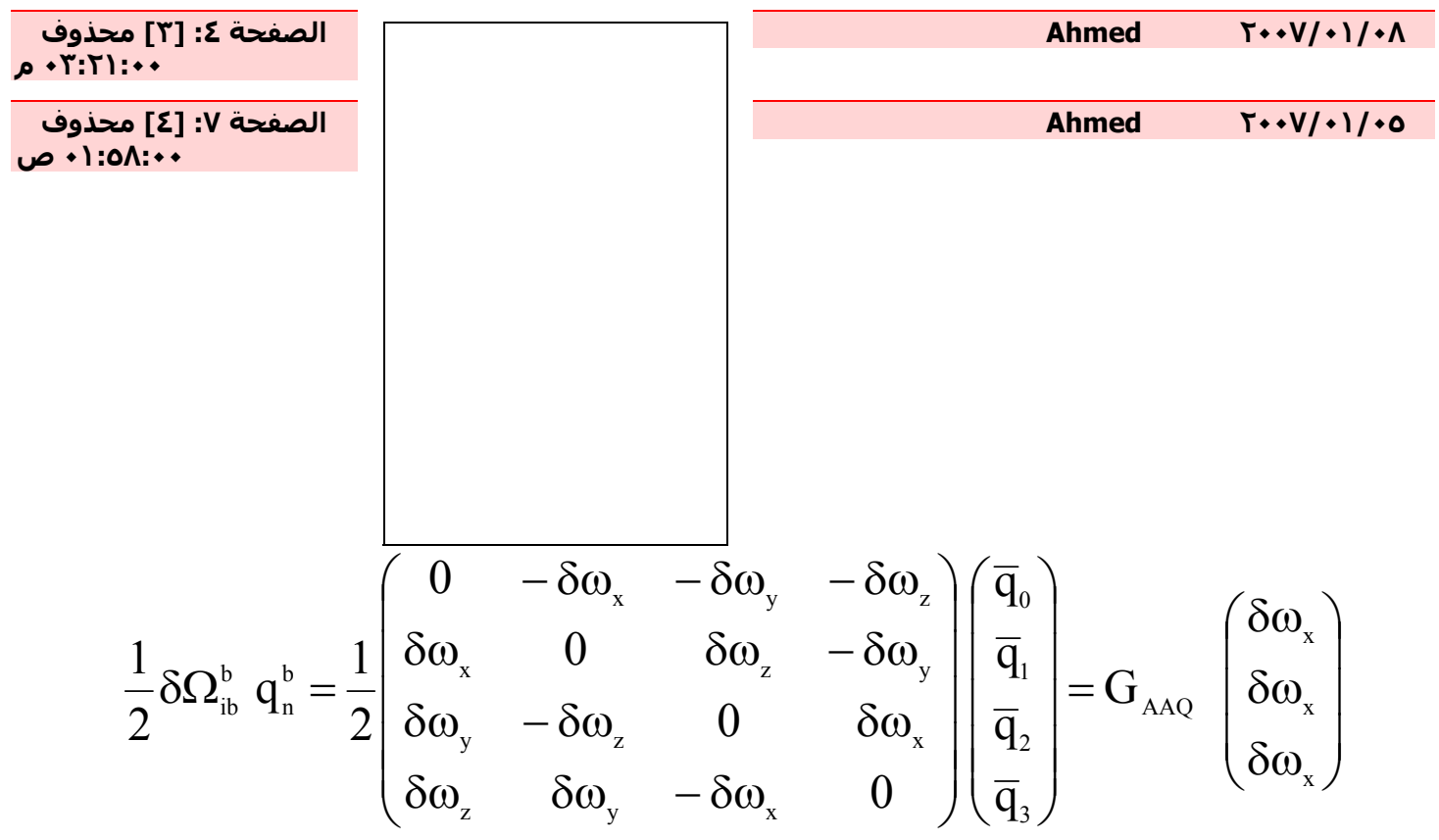

الصفحة r| (0) [0 منسّق

Ahmed

م $11: T V: \bullet r \cdot \bullet V / \cdot 1 / \cdot \varepsilon$

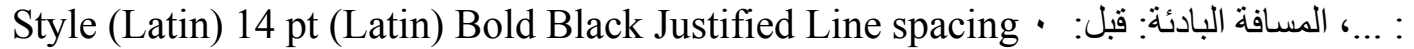
نقطة، السطر الأول: · نقطة 


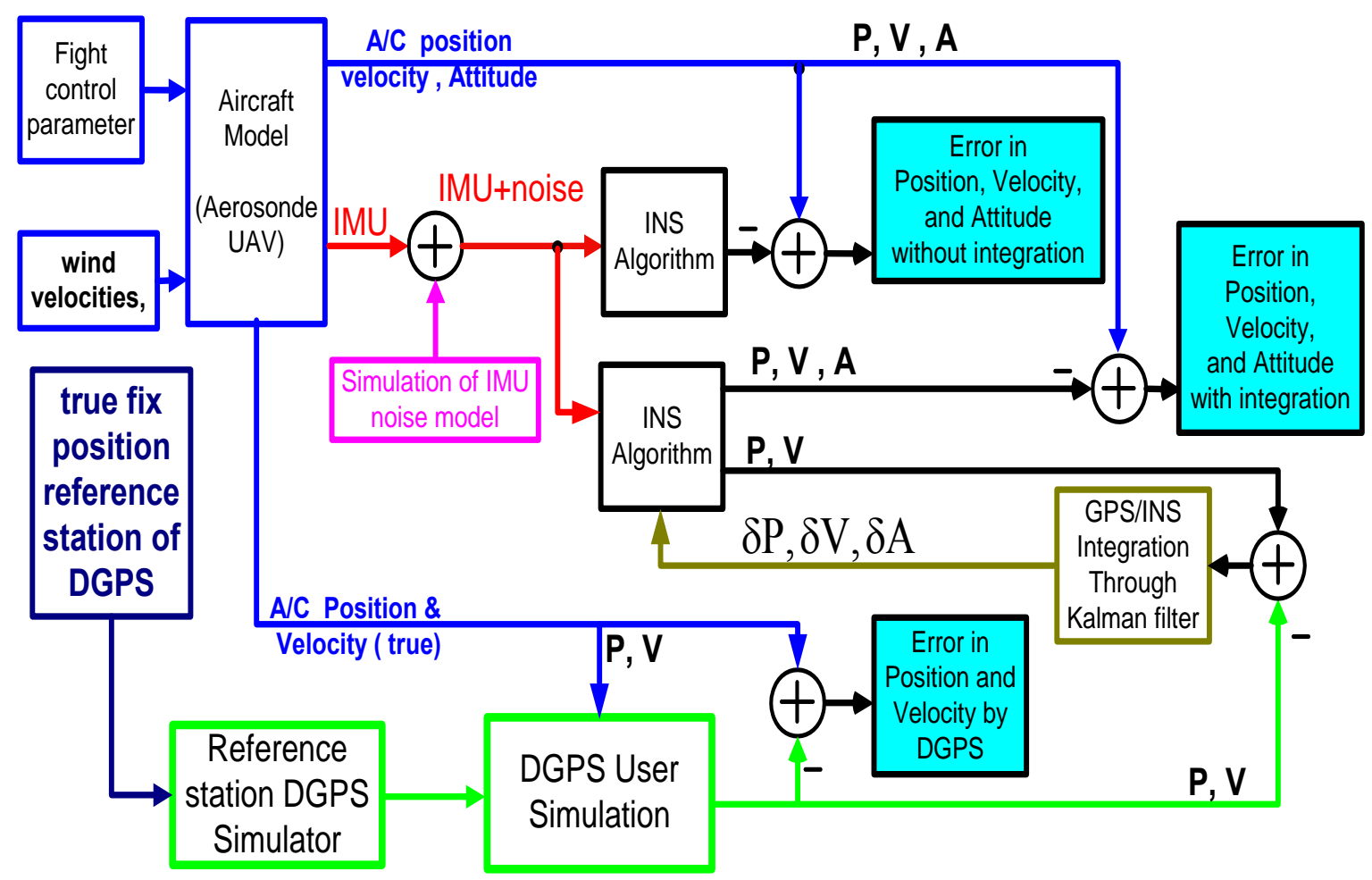

Fig. (4.4): Chart of simulation block diagram for test GPS/INS through Kalman integration

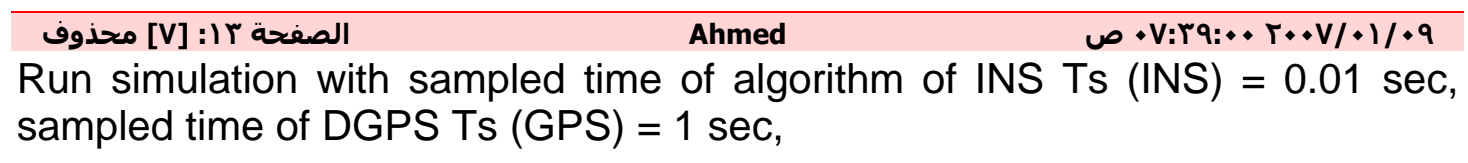

الصفحة با : [1] محذوف

Ahmed

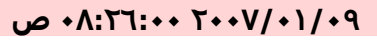

For test INS algorithm is using quaternions for attitude integration with GPS using different methods to solve navigation equation (Direct [GPS-D], Kalman [GPS-K],

\begin{tabular}{|c|c|}
\hline الصفحة Y | [ [9] محذوف & Ahmed \\
\hline
\end{tabular}

The INSQ is the less error than anther algorithm in roll $(\phi)$ less error until $10.0042 \mathrm{sec}$ from start, pitch $(\theta)$ less error until $78.0068 \mathrm{sec}$ from start, yaw $(\psi)$ less error until 10.0048 sec from start. 